\title{
Enediyne Antineoplastic Antibiotic
}

National Cancer Institute

\section{Source}

National Cancer Institute. Enediyne Antineoplastic Antibiotic. NCI Thesaurus. Code

C1927.

An enediyne antineoplastic antibiotic isolated from Streptomyces bacterial species or derived synthetically. After cycloaromatization, the enediyne core of these antibiotics is converted to a benzene diradical intermediate that removes hydrogens from deoxyriboses along the minor groove of DNA, resulting in single- and double-strand breaks and apoptosis. 